\title{
Force Distribution Analysis of Mechanochemically Reactive Dimethylcyclobutene
}

\author{
Wenjin Li, ${ }^{[a, b]}$ Scott A. Edwards, ${ }^{[c]}{ }^{2}$ Lanyuan Lu, ${ }^{[d]}$ Tomas Kubar, ${ }^{[\mathrm{e}]}$ Sandeep P. Patil, ${ }^{[\mathrm{f}]}$ \\ Helmut Grubmüller, ${ }^{[b]}$ Gerrit Groenhof, $^{*[b, g]}$ and Frauke Gräter*[a, f]
}

\begin{abstract}
Internal molecular forces can guide chemical reactions, yet are not straightforwardly accessible within a quantum mechanical description of the reacting molecules. Here, we present a force-matching force distribution analysis (FM-FDA) to analyze internal forces in molecules. We simulated the ring opening of trans-3,4-dimethylcyclobutene (tDCB) with on-the-fly semiempirical molecular dynamics. The self-consistent density functional tight binding (SCC-DFTB) method accurately described the force-dependent ring-opening kinetics of $t D C B$, showing quantitative agreement with both experimental and computational data at higher levels. Mechanical force was applied in two different ways, namely, externally by a constant pulling force and internally by embedding tDCB within a strained macrocycle-containing stiff stilbene. We analyzed the distribution of $\mathrm{tDCB}$ internal forces in the two different cases by FM-FDA and found that external force gave rise to a symmetric force distribution in the cyclobutene ring, which also scaled
\end{abstract}

linearly with the external force, indicating that the force distribution was uniquely determined by the symmetric architecture of $\mathrm{tDCB}$. In contrast, internal forces due to stiff stilbene resulted in an asymmetric force distribution within $t D C B$, which indicated a different geometry of force application and supported the important role of linkers in the mechanochemical reactivity of $\mathrm{tDCB}$. In addition, three coordinates were identified through which the distributed forces contributed most to rate acceleration. These coordinates are mostly parallel to the coordinate connecting the two $\mathrm{CH}_{3}$ termini of tDCB. Our results confirm previous observations that the linker outside of the reactive moiety, such as a stretched polymer or a macrocycle, affects its mechanochemical reactivity. We expect FM-FDA to be of wide use to understand and quantitatively predict mechanochemical reactivity, including the challenging cases of systems within strained macrocycles.

\section{Introduction}

Understanding the effect of mechanical forces on chemical reactions is an important challenge in modern chemistry and

[a] Dr. W. Li, Dr. F. Gräter

CAS-MPG Partner Institute and Key Laboratory for Computational Biology Shanghai Institutes for Biological Sciences Chinese Academy of Sciences (P.R. China)

[b] Dr. W. Li, Prof. H. Grubmüller, Dr. G. Groenhof Department of Theoretical and Computational Biophysics Max-Planck-Institute for Biophysical Chemistry Goettingen (Germany)

[c] Prof. S. A. Edwards

College of Physics and Technology, Shenzhen University 3688 Nanhai Ave, Shenzhen 518060, Guangdong (P.R. China)

[d] Prof. L. Lu

Division of Structural and Computational Biology School of Biological Sciences Nanyang Technological University (Singapore)

[e] Dr. T. Kubar Institute for Physical Chemistry Karlsruhe Institute of Technology (Germany)

[f] S. P. Patil, Dr. F. Gräter Heidelberg Institute for Theoretical Studies Heidelberg (Germany)

[g] Dr. G. Groenhof Department of Chemistry, Nanoscience Center University of Jyväskylä, Fl-40014 Jyväskylä (Finland)

Supporting information for this article is available on the WWW under http://dx.doi.org/10.1002/cphc.201300252. materials science. ${ }^{[1-5]}$ With increasing experimental advances, mechanical forces can be applied to targeted molecules by both ensemble and single-molecule methods, such as elongational flow, ${ }^{[6]}$ ultrasonic irradiation, ${ }^{[7-11]}$ single-molecule force clamp spectroscopy (SMFCS) ${ }^{[12-16]}$ molecular tensile machines with bottlebrush macromolecules, ${ }^{[17,18]}$ and molecular force probes. ${ }^{[4,19-22]}$ Recently, force-dependent structures and the kinetics of many chemical reactions have been intensively studied. $^{[1-4,15-18,23-31]}$

The analysis of force-altered structures and their reactivities is commonly based on the assumption that simple geometric parameters, such as the length of a bond, can serve as a reaction coordinate along which the force acts. However, force might distribute over several degrees of freedom in a more complex way. A crucial prerequisite for the understanding and prediction of mechanochemical events is therefore to reveal the coupling between the external force and individual degrees of freedom of the molecule, such as bonds or angles. Into which bond does the external force mainly propagate, and which one is most likely to break? Do internal forces in the bonds of the molecule change linearly with the externally applied force? Also, can we directly quantify forces in bonds in a strained molecule, as they occur, for example, in molecular force probes, to connect chemical reactivity to internal force? 
Boulatov and co-workers have developed a method to estimate the restoring force on a well-chosen internal coordinate based on the compliance matrix calculated for a Boltzmann ensemble of conformers. ${ }^{[22,29,32]}$ The restoring force, which can either result from an external force or from internal stress in macrocycles, quantifies the total amount of stretching force exerted on molecule along the chosen coordinate and it brings macrocycles and AFM results together appropriately. ${ }^{[33]}$ To gain more details on the forces distributed on each internal coordinate of the molecule, herein we resort to force distribution analysis (FDA) at atomic resolution. FDA based on molecular dynamics (MD) simulations has been developed to estimate the change in the internal forces of proteins upon applying an external load. ${ }^{[34]}$ It has been successfully applied to reveal force propagation in various protein domains of mechanical function, including the titin immunoglobulin 27 th domain, ${ }^{[34]}$ silk $\beta$ sheet crystals, ${ }^{[35]}$ and the von Willebrand factor A2 domain. ${ }^{[36]}$ FDA relies on the analysis of pairwise forces between atoms or groups of atoms as a measure for internal force, as opposed to forces acting on individual atoms or groups of atoms, which cancel out to zero over time. However, mechanochemical reactions require a quantum mechanical (QM) description, which, in contrast to MM force fields, does not implicate any definition of pairwise forces between atoms. To reveal the force distribution in quantum mechanically treated systems, we present herein a scheme to decompose the forces obtained from QM calculations onto interatomic connections of the reactant. Because the scheme combines force matching ${ }^{[37]}$ and force distri- bution analysis, we term our method force-matching FDA (FMFDA).

We applied FM-FDA to the ring opening of trans-3,4-dimethylcyclobutene (tDCB) as a model reaction. The effect of force on this reaction has been studied by embedding the reactive tDCB (Figure 1A) moiety into a molecular force probe, ${ }^{[19]}$ namely, macrocycles of varying sizes, which include a stiff stilbene for photoisomerization, two linkers, and tDCB (Figure 1B). The rate of ring opening increased when subjecting $T D C B$ to the internal force present in the macrocycles upon stilbene photoisomerization, with smaller macrocycles leading to higher rates. Theoretical studies have investigated how force alters the mechanism and potential energy surface of this mechanochemical reaction. ${ }^{[26,27]}$ Systems similar to tDCB, such as benzocyclobutene $e^{[9,23,24,26]}$ cyclopropane ${ }^{[16,32,38]}$ and cyclobutane, ${ }^{[28]}$ have been intensively investigated under mechanical force as well. Craig and co-workers have stretched and cleaved polycyclopropane with different polymer backbones, and showed that polynorbornene was more efficient in delivering force to the reactive moiety than polybutadiene; ${ }^{[16,33]}$ thus highlighting the non-negligible effect of the polymer backbone on the mechanical regulation of the reactive moiety. Such dependence on the polymer backbone was previously observed in a computational study on benzocyclobutene, for which the rupture force was dependent on the length of the attached polymer chains. ${ }^{[24,29]}$ Interestingly, a single structural parameter, namely, the distance between two $\mathrm{CH}_{3}$ termini, was found to be a good approximation of the reaction coordinate

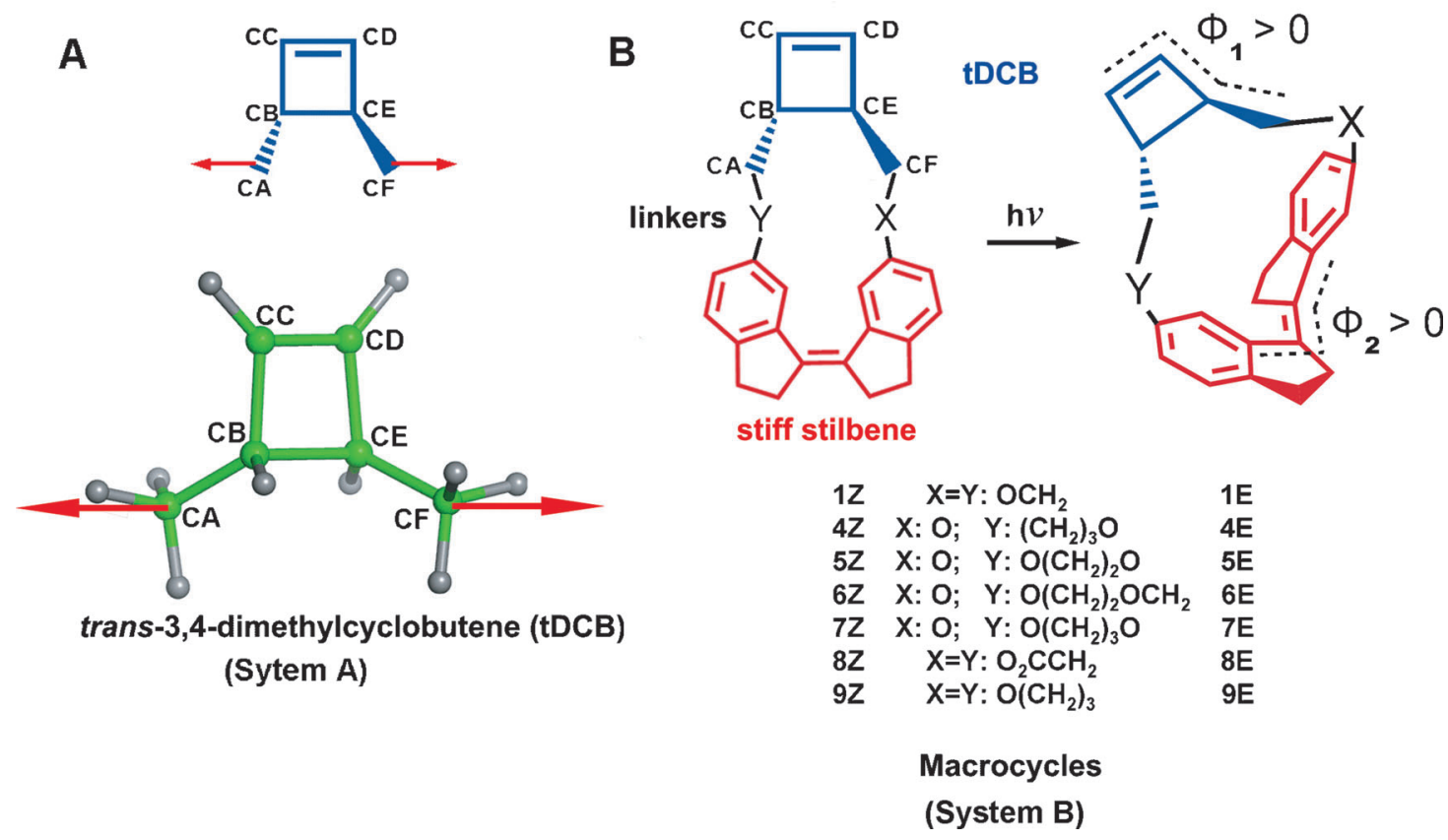

Figure 1. Two simulation systems to compare the effect of an external pulling force $(A)$ to that of internal stress (B): $A)$ tDCB under constant force pulling. Constant forces (red arrows) were applied to atoms CA and CF, which were the two carbon ends of tDCB. tDCB is shown schematically (upper panel) and as a three-dimensional structure (lower panel). B) tDCB (blue) was inserted into stiff stilbene (red) with two linkers (named $X$ and $Y$, black) to form a macrocycle. The $Z$ isomer of the macrocycles (left) can undergo photoisomerization and switch to the $E$ isomer (right). Different linkers, to vary the ring size of the macrocycles, were chosen as listed below. The set of macrocycles represents a subset of the previous experimental study. ${ }^{[19]} \mathbf{1 Z}$ is the $Z$ isomer of macrocycle $\mathbf{1}, 1 \mathrm{E}$ is the $E$ isomer (-) of macrocycle 1 , and so forth. In the $E$ isomer $(-)$, the dihedral angles $\Phi_{1}$ and $\Phi_{2}$ (indicated on the right) are of opposite signs. ${ }^{[19]}$ 
in the case of $\mathrm{tDCB}^{[19,29]}$ and cyclopropane. ${ }^{[16,30,32]}$ These results render tDCB a good model system to test FM-FDA.

It is becoming clear that the strained structure of the reactive moiety and its connecting lever arms collectively affect its mechanochemical reactivity. The collective effects may result in different patterns of force distribution within the reactive moiety. Therefore, we hypothesize that the patterns of force distribution in tDCB within strained macrocycles are different from those under external constant pulling forces, which is a common way to mimic molecular force probes in theoretical studies. ${ }^{[39-41]}$ Herein, we investigated the mechanochemical reactivity and underlying force distribution of tDCB when subjecting it to an internal force in a macrocycle (system $B$ in Figure 1) in comparison to an external constant pulling force (system A in Figure 1). We estimated force-dependent reaction rates for the two systems using conformational flooding, which is an enhanced sampling technique, ${ }^{[42,43]}$ and obtained rates in good agreement with experiments. By decomposing the effect of force on the chemical reaction onto individual bonds in $\mathrm{TDCB}$ by FM-FDA, we revealed that the cleaving bond was the primarily stressed connection in $\mathrm{TDCB}$, as opposed to a strong compression of the double bond on the opposite side of the four-membered ring; an effect that can be easily explained by simple structural mechanics. We found an external force to distribute into tDCB in a symmetric and linear manner, confirming that the pattern of force distribution was solely determined by the unique structural properties of the reactive moiety. The strained macrocycles resulted in an asymmetric force distribution pattern, highlighting the contribution from the lever arm, including the linkers, and possibly the stiff stilbene.

\section{Results and Discussion}

\subsection{Effective Forces on tDCB from Strained Macrocycles}

In molecular force probe experiments, the forces from the strained macrocycles are not available from experimental observations. Boulatov and co-workers calculated the restoring force either by the optimization of the macrocycles with cyclobutene removed, ${ }^{[19]}$ or from the relationship between the ensemble-averaged length of a local coordinate and the constant force. ${ }^{[32]}$ To take the dynamics of the system into account, herein we present an alternative way to determine the force from strained macrocycles. First, the relationship between the rate constant and the external force is built by estimating the rate under constant pulling force. Then, forces from strained macrocycles are estimated by comparing computed reaction rate constants in macrocycles to those of the linear pulling case. We note that the stilbene-containing macrocycle applies forces onto tDCB that most likely differ in their direction from the external force applied in opposite directions to the isolated tCDB (compare Figures $1 \mathrm{~A}$ and $\mathrm{B}$ ). Thus, the estimated force (we say effective force) represents a horizontal force on the tDCB methyl group that is needed to replace the-supposedly complex-force or stress within the macrocycle such that the same acceleration is achieved. In the following, we refer to "stress" instead of "force" within the molecule, in analogy to the engineering stress used in structural machines, even though stress here is given in units of force because normalization by area is ill-defined in a molecular structure.

\subsubsection{Force-Rate Curve Under Conformational Flooding}

Ring opening of $\mathrm{tDCB}$ is very slow and cannot be simulated by standard MD simulations. Conformational flooding provides a method to accelerate the transition of chemical reactions without prior knowledge of the mechanism of the reaction. ${ }^{[42,43]}$ Herein, we applied a constant flooding potential, which was built on a $1 \mathrm{~ns}$ equilibrium simulation of tDCB at the self-consistent-charges density-functional tight-binding $(\mathrm{SCC}-\mathrm{DFTB})^{[44]}$ level without pulling. With an effective flooding potential of $130 \mathrm{~kJ} \mathrm{~mol}^{-1}$, the rate of the ring opening of tDCB was accelerated to be accessible within the timescale of our simulations. We performed 500 to 4000 simulations (depending on the external force, see Table S1 in the Supporting Information), each $50 \mathrm{ps}$ long, in the presence of the flooding potential under pulling forces of $0,100,200,300,400,500,600$, and $700 \mathrm{pN}$. The rate constant was then estimated from the first passage times (see the Supporting Information). The estimated rates under different forces are shown in Figure 2A (see also Table S1).

As expected, reaction rates increase with force. However, at large forces beyond $500 \mathrm{pN}$, the results diverge from a simple exponential increase of the rates with the pulling force, with the impact of the pulling force becoming steadily smaller. As proposed by Hummer and co-workers, ${ }^{[45]}$ the force-dependent rate constant was given by Equation (1):

$$
\begin{aligned}
& r(F)= \\
& r_{0}\left(1-v F \Delta x_{\mathrm{r}} / \Delta G^{\ddagger}\right)^{1 / v-1} \exp \left\{\beta \Delta G^{\ddagger}\left[1-\left(1-v F \Delta x_{\mathrm{r}} / \Delta G^{\ddagger}\right)^{1 / v}\right]\right\}
\end{aligned}
$$

in which $\beta=1 / k_{\mathrm{B}} T, r_{0}$ is the rate constant without external force, $F$ is the external force, $\Delta G^{*}$ is the free energy of activation, $\Delta x_{r}$ is the distance between reactant state (RS) and transition state (TS) along the pulling coordinate, and $v$ is a parameter that characterizes the shape of the free-energy profile: $v=$ $1 / 2$ corresponds to a cusp free energy surface. ${ }^{[45,46]}$ Taking $v=$ $1 / 2$, Equation (1) fits to the results very well (solid line in Figure $2 \mathrm{~A}$ ). $\Delta x_{r}$ is estimated from the fit to be $0.62 \AA$. This is consistent with the difference in the CA-CF (we refer to the atoms in $\mathrm{tDCB}$ according to the labels shown in Figure $1 \mathrm{~A}$ ) bond length between the RS and TS obtained in another theoretical study, with $\Delta x_{r}$ of $0.74 \AA^{[19]}$ (Table S2).

For the ring opening of $t D C B$ under constant flooding potential, we observed a concerted conrotatory mechanism (Figure S11), as reported in the study of cyclobutene ${ }^{[26,47]}$ and benzocyclobutene under trans pulling. ${ }^{[23]} \mathrm{A}$ typical example of a trajectory is given in Figure S1. The potential energy along the CB-CE bond at the SCC-DFTB level was estimated to be $31.1 \mathrm{kcal} \mathrm{mol}^{-1}$, which was comparable to the energy barrier of $27.0 \mathrm{kcal} \mathrm{mol}^{-1}$ estimated at the MP2/6-31+G level (see Fig- 
A

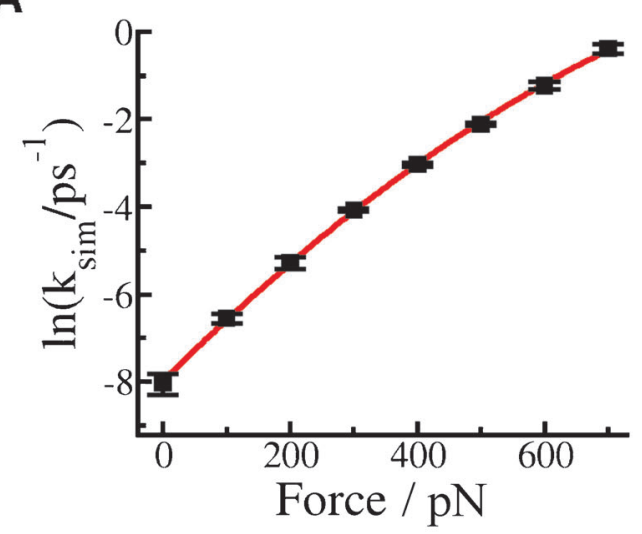

B

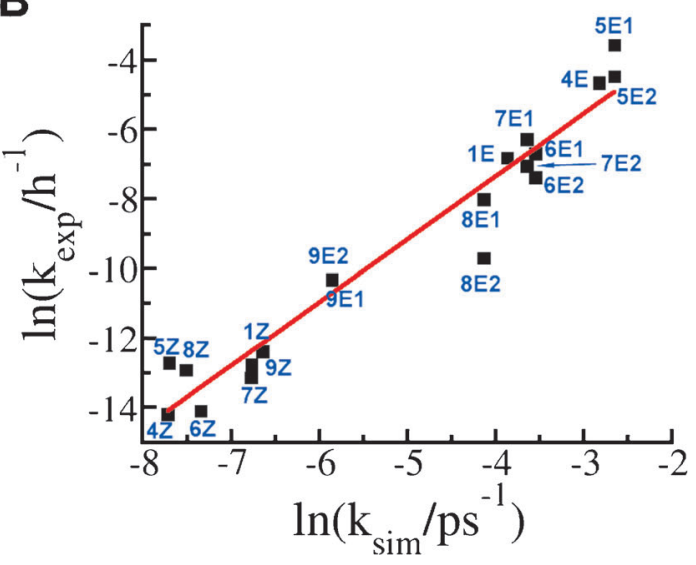

Figure 2. Force-dependent reaction rates of the ring opening of $t D C B$ : A) The rate constants estimated herein are the rates for the ring opening of tDCB with an effective flooding potential of $130 \mathrm{~kJ} \mathrm{~mol}^{-1}$. Three rate constants were obtained by dividing the simulations into three parts, and the average rate and standard deviation are shown in black. Red: fit to the Dudko-Hummer model. B) The logarithm of rates estimated from conformational flooding have a linear relationship with experimental ones (red line). The labels indicate the corresponding macrocycles (Supplementary Table 9 in ref. [19]). For example, $\mathbf{1 Z}$ and $\mathbf{1 E}$ are the $Z$ and $E$ isomers (-) of macrocycles $1 ; 5 \mathrm{E} 1$ and $\mathbf{5 E} 2$ are diastereomers 1 and 2 of the $E$ isomer of macrocycle 5 .

ure S2) and agreed well with the energy barrier of $31 \mathrm{kcal}$ $\mathrm{mol}^{-1}$ reported in ref. [19]. The distance between TS and RS along the $\mathrm{CB}-\mathrm{CE}$ bond were 0.54 and $0.56 \AA$ at the SCC-DFTB and MP2/6-31 + G level, respectively (see Table S13). Both were consistent with the corresponding value of $0.56 \AA$ reported in ref. [19] (Table S2). These results demonstrate the accuracy of the SCC-DFTB method for the reaction studied herein.

\subsubsection{Effective Force on $t D C B$}

Next, we used the force-rate relationship obtained above (Figure $2 \mathrm{~A}$ ) to estimate the effective forces that are added on tDCB from the strained macrocycles using the rate of ring opening of $t D C B$ in the macrocycle. This requires knowledge of the reaction rates of $t D C B$ opening for different macrocycle sizes, which we obtained using again MD simulations and conformational flooding. We applied the same flooding potential of $130 \mathrm{~kJ} \mathrm{~mol}^{-1}$ as that obtained for the pulled tDCB and esti-
Table 1. Mechanochemical reactivity of macrocycles. The rate of ring opening, $k$, and the corresponding effective force obtained from comparison to rates upon pulling $t D C B$ are given.

\begin{tabular}{lllr|} 
Macrocycles $^{[a]}$ & $k\left[\mathrm{ps}^{-1}\right]$ & STD $^{[\mathrm{b}]}$ & Effective force $[\mathrm{pN}]$ \\
\hline $\mathbf{1 Z}$ & $1.31 \times 10^{-3}$ & $1.60 \times 10^{-4}$ & 96 \\
$\mathbf{1 E}$ & $2.09 \times 10^{-2}$ & $1.05 \times 10^{-3}$ & 322 \\
$\mathbf{4 Z}$ & $4.45 \times 10^{-4}$ & $9.30 \times 10^{-5}$ & 19 \\
$\mathbf{4 E}$ & $5.94 \times 10^{-2}$ & $2.32 \times 10^{-3}$ & 420 \\
$\mathbf{5 Z}$ & $4.51 \times 10^{-4}$ & $8.60 \times 10^{-5}$ & 19 \\
$\mathbf{5 E}$ & $7.05 \times 10^{-2}$ & $3.00 \times 10^{-3}$ & 438 \\
$\mathbf{6 Z}$ & $6.47 \times 10^{-4}$ & $1.26 \times 10^{-4}$ & 44 \\
$\mathbf{6 E}$ & $2.89 \times 10^{-2}$ & $1.01 \times 10^{-3}$ & 351 \\
$\mathbf{7 Z}$ & $1.14 \times 10^{-3}$ & $2.70 \times 10^{-5}$ & 86 \\
$\mathbf{7 E}$ & $2.62 \times 10^{-2}$ & $1.20 \times 10^{-3}$ & 342 \\
$\mathbf{8 Z}$ & $5.46 \times 10^{-4}$ & $8.80 \times 10^{-5}$ & 33 \\
$\mathbf{8 E}$ & $1.60 \times 10^{-2}$ & $8.69 \times 10^{-4}$ & 298 \\
$\mathbf{9 Z}$ & $1.15 \times 10^{-3}$ & $1.15 \times 10^{-4}$ & 87 \\
$\mathbf{9 E}$ & $2.87 \times 10^{-3}$ & $2.42 \times 10^{-4}$ & 156 \\
\hline [a] The names of the macrocycles refer to Figure $1 \mathrm{~B}$. [b] STD=Standard \\
Deviation. \\
\hline
\end{tabular}

mated the rate constant from first passage times observed in 1000 to 4000 simulations (Table S3). The estimated rates are shown in Table 1.

By looking up the forces corresponding to the rates for the macrocycles in Figure $2 \mathrm{~A}$, we obtained values for effective forces that were exerted onto the two ends of the $t D C B$ part within different macrocycles; these are shown in Table 1. Because the reaction rate in a macrocycle reflected the collective mechanochemical effect from the strained structure of $t D C B$, the linkers, and the stiff stilbene, the effective force, which was derived from this reaction rate, quantified an apparent external force from the environment exerted on the two termini of $t D C B$. It was recently demonstrated that the relationship between the intrinsic force or restoring force on a properly chosen internal coordinate and the kinetic stability of the reactive site was independent of the way of developing the restoring force, for example, constant force pulling or internal stress in macrocycles. ${ }^{[29,32,33]}$ This rationalizes our approach to derive the effective force from the force-rate curve. Herein, we take the coordinate along the $\mathrm{CA}-\mathrm{CF}$ bond as the pulling coordinate, analogously to the route taken in ref. [19], so that the effective force should be comparable to the restoring force. Indeed, they are highly correlated (Figure S13). One possible reason for quantitative differences could be lower accuracy of the semiempirical method we used; however, it was sufficient for our purpose of deriving the effective force to assess the linearity of the distributed forces.

Similar to isolated tDCB, in the strained macrocycle we also observed ring opening along a concerted conrotatory mechanism (Figure S11). Thus, the reaction coordinates of the two systems are similar, which further justifies our approach to map effective forces to stress in macrocycles.

Clearly, a reliable estimate of the effective force requires accurately estimating the reaction rate in the macrocycles. To validate our rate estimations, we examined the correlation between the rates estimated with the flooding potential and the 
experimentally determined rates in the stilbene-linked $t D C B$ macrocycles. The experimental rates after extrapolation to $300 \mathrm{~K}$ by using Eyring's equation (see the Supporting Information) ${ }^{[19]}$ are shown against the computed rates in Figure $2 \mathrm{~B}$. Because the computed rates were obtained under a flooding potential, we would not expect the computed and experimental rates to be equal. As shown in Figure $2 \mathrm{~B}$, the experimental rate for macrocycle $4 Z$ was $\ln \left(k_{\exp } \mathrm{h}^{-1}\right)=-14.2$, whereas the computed one was $\ln \left(k_{\text {sim }} \mathrm{ps}^{-1}\right)=-7.7$. The difference corresponds to an energy difference of $105 \mathrm{~kJ} \mathrm{~mol}^{-1}$, which is comparable to the reduction in the free energy barrier $\left(115 \mathrm{~kJ} \mathrm{~mol}^{-1}\right)$ due to the flooding potential (see the Supporting Information); this validates the computed rates obtained with conformational flooding. In addition, the computed and experimental rates correlated strongly with each other. We found a slope of 1.8 instead of 1 for the linear fit in Figure $2 \mathrm{~B}$. In fact, the application of the flooding potential alters the potential energy surface at the RS. We found that the same flooding potential differently distorted the free energy landscape at different pulling forces (Figure S12), which could be one possible reason for the observed discrepancy. Despite this discrepancy, regarding the satisfying overall agreement with experiment, we assume in the following that the effective force is a valid measure for the mechanical effect at play in macrocycle system $B$.

\subsection{Linear Response of Cyclobutene to an External Force}

How does the force distribute within tDCB when it is subjected either to constant pulling force or within the stilbene-containing macrocycles? Are there differences in the way force distributes into tDCB when pulled with an external force, compared with being inserted into the strained macrocycles? The study of force distribution in tDCB under forces applied in these two different ways may shed some light on the abovementioned questions.

The macrocycles subject cyclobutene to a pulling force that depends on the size and structure of the macrocycle. For comparison to this rather complex way of force application, we first simplified the system by directly pulling tDCB at the methyl groups in opposing directions by constant forces ranging from 0 to $600 \mathrm{pN}$ (Figure $1 \mathrm{~A}$ ). For each pulling force, we ran ten simulations with different starting structures at the SCC-DFTB level, each of which was 2 ns long. To analyze the distribution in internal stresses, we applied the newly developed FM-FDA (see the Computational Methods section) to tDCB under pulling forces. To this end, first, a pairwise force field (PWFF) was constructed on the SCC-DFTB simulations at $0 \mathrm{pN}$ by force matching (for details, see the Supporting Information). Based on the PWFF, the force distribution in $\mathrm{TDCB}$ was then estimated for each force and is shown for $400 \mathrm{pN}$ in Figure $3 \mathrm{~A}$ (all data are given in Figure S3 and Table S4 in the Supporting Information). To see how this distribution scales with force, typical pairwise forces as a function of external force are shown in Figure 4A. For comparison, we also obtained the force distribution within TDCB at the O3LYP/6-311G $(2 d, p)$ level and found it to be in quantitative agreement with results ob-

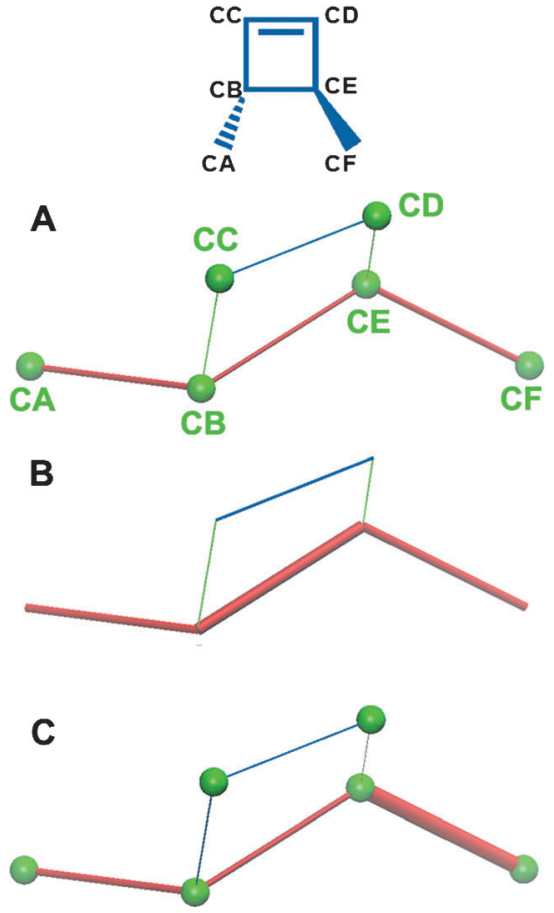

Figure 3. Force distribution in $t D C B$ : Top: Schematic structure of tDCB. A) Force distribution in tDCB under a constant force of $400 \mathrm{pN}$. B) Force distribution (axial stresses) in a mechanics model that mimics tDCB composed of elastic beams under a constant force obtained from finite-element analysis. C) Force distribution in tDCB within the $E$ isomer (-) of macrocycle 4. Pairwise forces are represented by cylinders connecting atom pairs. The radius and the color of the cylinders represent the magnitude and direction of the force, with blue for repulsive and red for attractive forces. Forces are normalized by the constant pulling force (A and B) or the effective force (C). Only the force distribution within the carbon backbone of $t D C B$ is shown. Green atoms are the carbon atoms in tDCB and are labeled in the same way as described in Figure $1 \mathrm{~A}$.

tained at the SCC-DFTB level (Figure 4A and Table S5); this again demonstrated that $\mathrm{TDCB}$ could be described accurately at the SCC-DFTB level.

As shown in Figure 4A, all pairwise forces scale linearly with the external force, with correlation coefficients of about one (Table S6) for all bonds. In addition, the force distribution is largely symmetric, with two equivalent bonds in tCDB carrying highly similar forces (Figure 5A). As expected, the largest forces were carried by the bonds between the methyl groups and the cyclobutene groups (CA-CB and $C E-C F$, with $\approx 50 \%$ of the external force), onto which the pulling force directly applied. These were closely followed by the cleaving bond in the tDCB ring, $C B-C E$, which carried roughly $30 \%$ of the external force, in accordance with the chemical ring structure, which could, similar to parallel springs, distribute the external force more widely. Interestingly, one bond in the cyclobutene ring is compressed, namely, the CC-CD bond, in contrast to the other bonds. Compression increases linearly with the applied force, with a compressive force of about $15 \%$ of the external force.

We next tested whether this force distribution pattern, most importantly the compression of the upper CC-CD bond, could be explained by simple structural mechanics. To this end, we constructed a three-dimensional structure of the tDCB mole- 


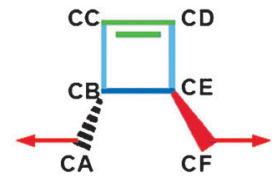

A

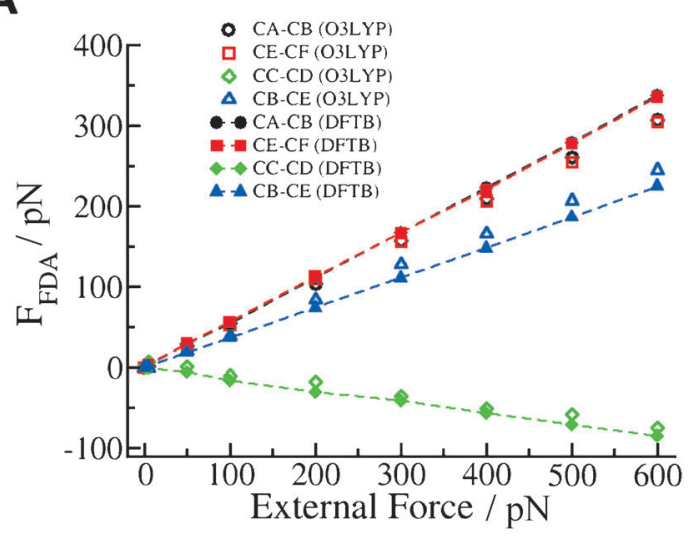

B
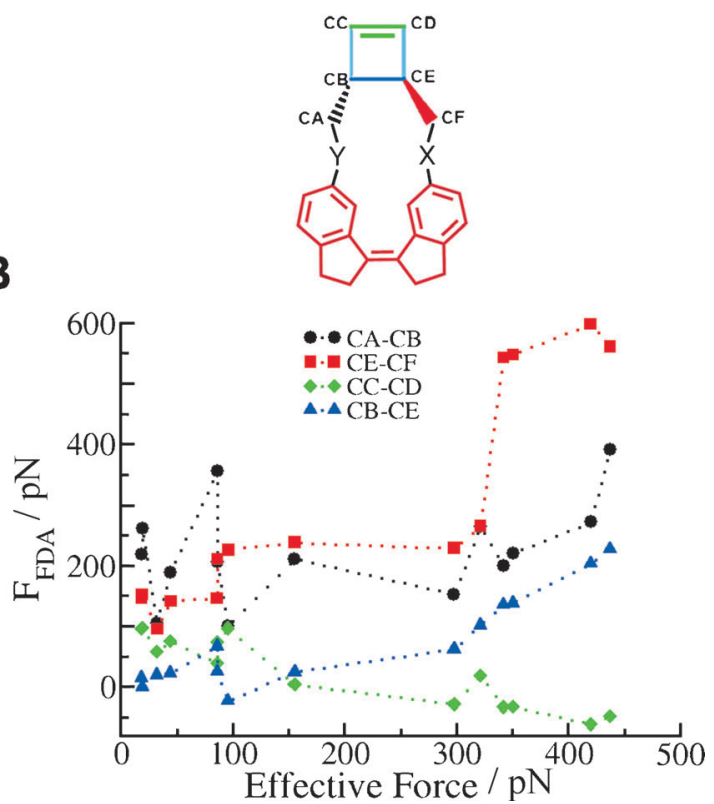

Figure 4. Force distribution of tDCB under varying forces: $A$ ) and $B$ ) show the distributed forces on four representative bonds in systems $A$ and $B$ (Figure 1), respectively. Top: Schematic structures of these two systems are shown. Bonds are colored the same as the corresponding data below. A) Force distributions estimated at the SCC-DFTB level (filled symbols) and the ones at the O3LYP/6-311G $(2 d, p)$ level (open symbols with a dashed line) are consistent. The forces on individual bonds scale linearly with external forces. B) Effective forces in macrocycles were estimated based on their rate of ring opening compared with external pulling forces (red curve in Figure $2 \mathrm{~A}$ ). Forces on individual bonds do not linearly scale with the external force.

cule from single beams for each chemical bond with equal stiffness for each bond and subjected this molecular structure to a constant force at the beam ends that corresponded to the methyl groups (see the Supporting Information). We qualitatively obtained beam stresses directly comparable to those from FM-FDA, including compression in the CC-CD bond, in
A

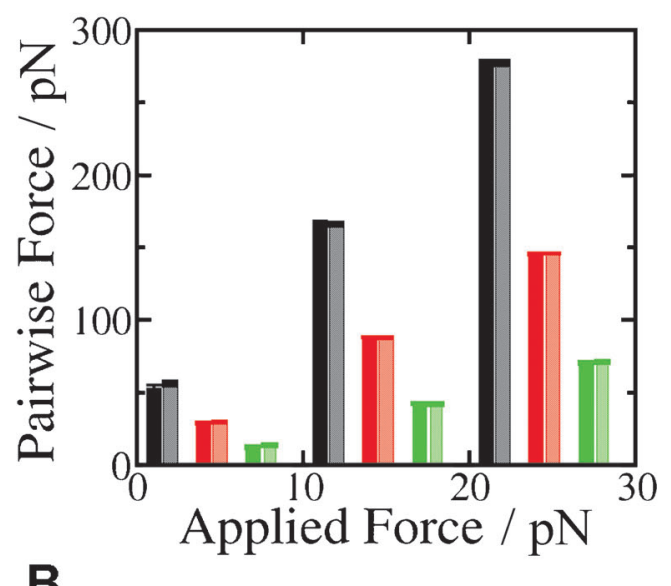

B

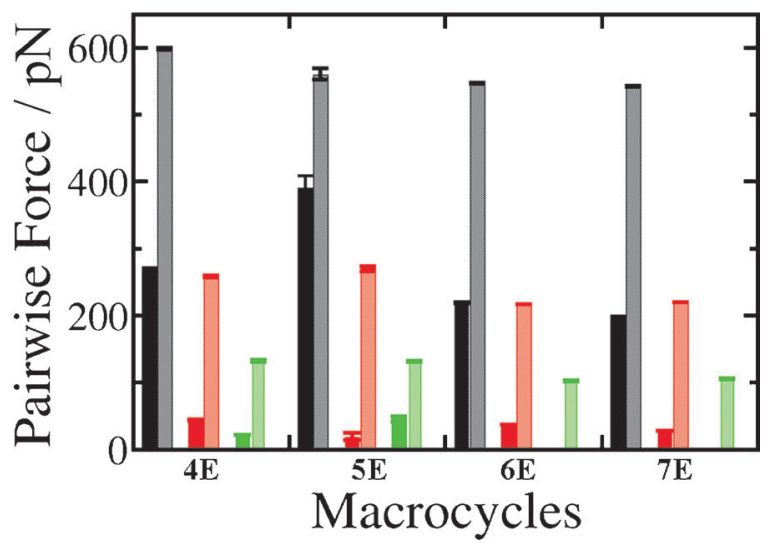

Figure 5. Comparison of forces on symmetric bonds: $A)$ and $B$ ) show the forces in three pairs of symmetric bonds; $C A-C B$ (black) and CE-CF (grey), CA-CD (dark red) and CB-CF (light red), and CB-CD (dark green) and CC-CE (light green). In the case of system A (Figure 1), the forces in the three pairs of bond are equal, whereas in the case of system $B$, for the strained $E$-isomer macrocycles, the forces on these bonds largely vary in any of the macrocycles tested.

contrast to tensile stresses in all other connections (compare the structures in Figure $3 \mathrm{~A}$ and $\mathrm{B}$ ). Similar patterns were observed when the methyl groups (CA and CF) were located in the plane of the cyclobutene ring or in a cis position. All internal forces scale linearly with the external force, similar to those in our quantum mechanics calculations. Thus, force distribution within $\mathrm{tDCB}$ can, to a large extent, be explained by a simple mechanical model. Overall, despite the highly nonlinear QM system under consideration, tCDB behaves under mechanical stress similar to an analogous structure of elastic beams, that is, Hookean springs.

\subsection{Asymmetric Stress in Stiff Macrocycles}

As demonstrated in several studies on cyclopropane ${ }^{[16]}$ benzocyclobutene, ${ }^{[24]}$ and cyclobutenes $^{[29]}$ the connecting polymer backbone affected the forces exerted on the reactive moiety. We thus expected a different force distribution pattern in tDCB in macrocycles due to the different size and geometry of the linkers. To test this expectation, we determined the individual 
forces in bonds in $\mathrm{tDCB}$ when inserted into different macrocycles. To this end, analogous to isolated and pulled tDCB, we ran ten simulations of all macrocycles, with each simulation lasting $1 \mathrm{~ns}$. Using the same PWFF built for $\mathrm{tDCB}$, we obtained force distributions within the $T D C B$ moiety of the macrocycles. The distribution in the $E$ isomer (-) of macrocycle 4 is mapped onto the tDCB structure in Figure $3 C$, and distributed forces on representative bonds in macrocycles 4, 5, 6, and 7 are shown in Figure 5B (the results are summarized in Tables S7 and S8 and Figure S4). Although standard errors of the mean of internal forces suggest convergence of our results, we cannot exclude the possibility that conformational space is overlooked by our sampling scheme. In contrast to externally applied forces, which lead to identical forces in chemically equivalent pairs of bonds due to the symmetry of $t D C B$, now the forces in equivalent bonds differ greatly, resulting in an asymmetric force distribution pattern (compare Figure $5 \mathrm{~A}$ and $B)$. For example, the force on bond CE-CF is much larger than that on the chemically equivalent bond $C A-C B$. Because the linker attached to bond $\mathrm{CE}-\mathrm{CF}$ in the macrocycle is shorter than the linker on the other side, we suggest that the flexibility of the linker (or its spring constant) affects the force on the bond. This asymmetry in the force distribution was confirmed when we used the cis macrocycle as a reference state to calculate the PWFF, which was then used to estimate internal stress in the respective trans state.

Another remarkable consequence of asymmetry is that individual forces in bonds can reach $600 \mathrm{pN}$, as in the CE-CF bond of the $4 \mathrm{E}$ isomer, which has an effective force of about $450 \mathrm{pN}$. In contrast, a force of only $300 \mathrm{pN}$ was reached in the same bond at a very similar external force of $500 \mathrm{pN}$. Thus, even though the small and highly strained $4 \mathrm{E}$ macrocycle gives rise to partial high-bonding forces in tDCB, the force asymmetry and force concentration do not yield correspondingly high rates, possibly because other reactive bonds, most importantly the cleaving $\mathrm{CB}-\mathrm{CE}$ bond, is under comparably low stress (see below).

Given the significance of asymmetry, we expected linear scaling of the internal forces in the chemical bonds of $t D C B$ observed for external pulling (Figure $4 \mathrm{~A}$ ) to be abrogated, at least partially, in the strained macrocycles (Figure 4B). Indeed, the perfect linearity seen for pulling tDCB (Figure $4 A$ ) has vanished to a large extent, with the largest correlation coefficients of 0.89 and 0.87 found for $C E-C F$ and $C B-C E$, respectively (Table S6). Even tDCB embedded into macrocycles with stiff stilbene as a $Z$ isomer, that is, the supposedly unstrained state, is under some residual internal stress. For example, in the case of the $Z$ isomer of macrocycle 4 (4Z), with an effective force of only about $20 \mathrm{pN}$, the CA-CB bond exhibits a force as high as $220 \mathrm{pN}$. Overall, pulling a reactive moiety such as tDCB by a stiff stilbene is significantly different from a constant force pulling along a predefined direction because the macrocycles give rise to an observable asymmetric and nonlinear force distribution, even for the supposedly relaxed $Z$ isomers. These results confirmed that the linkers and stiff stilbene established a complex pulling geometry, the effect of which could be quantified by FDA, and supported previous observations ${ }^{[16,24,29]}$ that parts outside of the reactive moiety crucially affected the mechanochemical reactivity of the molecule.

Given the remarkably different force distribution observed in the mechanoreactive moiety embedded in the strained macrocycle, as opposed to pulling the same moiety with an external force, our findings would predict that these two ways of force application result in different force-dependent kinetics. However, in the case of cyclobutene, experimental rates are only at hand for the strained macrocycles, but are not available from ultrasound experiments of the same molecule. AFM experiments of force-induced tDCB ring opening would be able to fill this gap.

\subsection{Contribution of Stressed Bonds to Increased Reaction Rates}

Mechanical work added to the system by pulling can be determined by $F \Delta x_{r}$ in which $\Delta x_{r}$ is the change in length along the reaction coordinate upon the formation of the TS [compare with Eq. (1)]. Because the length of the CA-CF bond was reported to be a good approximation of the reaction coordinate, ${ }^{[16,19,29,30,32]}$ we defined the change in the CA-CF bond length between RS and TS as $\Delta x_{r}$ How does the stress in individual bonds, as observed by FM-FDA, contribute to the overall mechanical work?

We estimated the length difference between RS and TS, $\Delta x_{\mathrm{r}}^{\mathrm{ij}}$, for all pairwise bonds between atoms $i$ and $j$ within the carbon backbone of tDCB, that is, the six carbon atoms, for all macrocycles, and then averaged this over the macrocycles (Table S2). RS and TS structures were obtained from optimizations at the O3LYP level. ${ }^{[19]}$ The mechanical work per bond between atoms $\mathrm{i}$ and $\mathrm{j}$ is then given by $F_{\mathrm{FDA}}^{\mathrm{ij}} \Delta x_{\mathrm{r}}^{\mathrm{ij}}$, in which $F_{\mathrm{FDA}}^{\mathrm{ij}}$ is the force distributed onto this bond. We identified a combination of three bonds that captured most of the mechanical work, leading to an acceleration of ring opening [Eq. (2)]:

$F_{\mathrm{ext}} \Delta x \approx F_{\mathrm{FDA}}^{\mathrm{CA}-\mathrm{CE}} \Delta x_{\mathrm{r}}^{\mathrm{CA}-\mathrm{CE}}+F_{\mathrm{FDA}}^{\mathrm{CB}-\mathrm{CE}} \Delta x_{\mathrm{r}}^{\mathrm{CB}-\mathrm{CE}}+F_{\mathrm{FDA}}^{\mathrm{CB}-\mathrm{CF}} \Delta x_{\mathrm{r}}^{\mathrm{CB}-\mathrm{CF}}$

in which $F_{\text {ext }} \Delta x_{\mathrm{r}}$ is the total work. Figure 6 shows the actual total work added to the system by pulling with $F_{\text {ext }}$ in comparison to the sum over three bonds, according to Equation (2), both for the different macrocycles and for tDCB under different external forces. These results are strongly correlated with a correlation coefficient of 0.9 (see the Supporting Information).

Thus, the external work is mainly distributed onto the bonds of $C A-C E, C B-C E$, and $C B-C F$. In other words, only the forces on these three bonds contribute significantly to the acceleration of the reaction rate. Meanwhile, these bonds undergo great change from RS to TS (see Table S2), indicating their relevance in the reaction mechanism. Remarkably, these three coordinates are all along the coordinate of the CA-CF bond, which is a good reaction coordinate, according to previous reports. ${ }^{[19,29]}$ Clearly, the CB-CE bond is vital to the reaction because its cleavage leads to the product. The two symmetric bonds of $C A-C E$ and $C B-C F$ open with $C B-C E$ in a correlated manner and are also involved in rotation around $C B-C E$ upon 


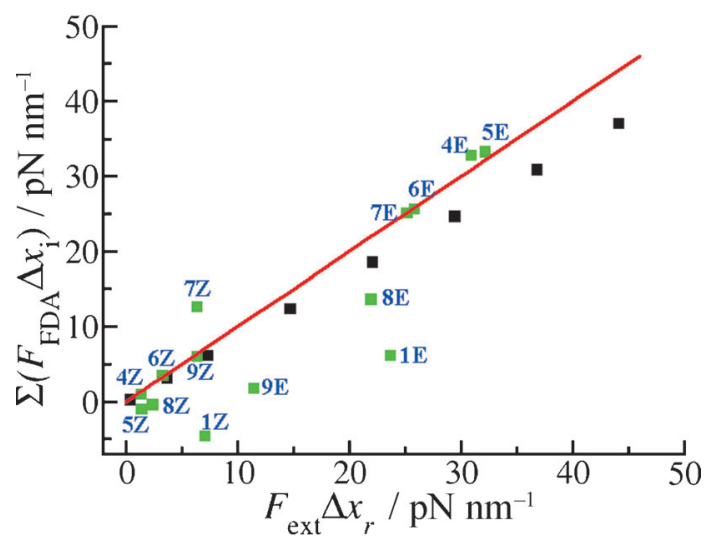

Figure 6. Decomposition of the mechanical work applied to tDCB to enhance its mechanochemical reaction: $F_{\text {ext }} \Delta x_{\mathrm{r}}$ is the total effect of the external force on the reaction [left part of Eq. (2)]. $\Sigma\left(F_{\mathrm{FDA}} \Delta x_{i}\right)$ is the sum over the effect of force on bonds $C A-C E, C B-C E$, and $C B-C F$ [right part of Eq. (2)]. The black and the green squares show the data from simulations of system$S A$ and $B$ (Figure 1), respectively. The red line indicates equality of the total work and the total decomposed contributions.

ring opening (Figure S2). This renders all three bonds relevant to the reaction. In the case of pulling tDCB by constant forces, $\mathrm{CA}-\mathrm{CE}, \mathrm{CB}-\mathrm{CE}$, and $\mathrm{CB}-\mathrm{CF}$ contribute about 28,29 , and $28 \%$, respectively, to the total mechanical work. In the case of the macrocycle, this symmetry is again abrogated because the bond connected to the rigid linkers (e.g. CB-CF) contributes more mechanical work than the bond connected to the soft linkers (e.g. CA-CE); this indicates significant impact of the linkers on force transduction. ${ }^{[16]}$

\section{Conclusions}

To investigate the mechanical properties of a single bond in a chemical reaction, a method to perform force distribution analyses in QM systems, termed FM-FDA, has been developed and tested on the ring opening of $t D C B$. We investigated the effect of force on the ring opening of this cyclobutene under two different conditions, constant force pulling and pulling within macrocycles, by using QM simulations at the SCC-DFTB level. For a direct comparison to the experimental results, the ring-opening rates of $\mathrm{tDCB}$ within macrocycles were estimated by conformational flooding simulations. The effective forces present in the macrocycles upon photoisomerization of stiff stilbene were then estimated on the basis of a calibrated rateforce relationship. As an important validation of the developed FM-FDA, our analysis with FM-FDA agrees with and explains some conclusions from previous AFM or molecular force probe experiments and computational studies on tDCB itself and its analogues. ${ }^{[16,19,24,29,32]}$ In addition, our analysis could provide complementary information at the single-bond level.

For computational studies on mechanochemistry, constrained geometries simulate external force (COGEF)- and "external force is explicitly included" (EFEI)-based simulations are two theoretical approaches commonly used.$^{[48,24]}$ They have proven to be powerful in quantifying the potential energy profile under external force, and important structures, including the
RS, TS, product state (PS), and intermediates. ${ }^{[5,11,24,26]}$ The compliance-based method to estimate the restoring force along a chosen coordinate quantifies the overall force on the reactive molecule and is very powerful in its own right, ${ }^{[22,29,32]}$ and we consider the FM-FDA method developed herein to be a useful complementary tool because it resolves the distribution pattern of internal pairwise forces and provides the possibility of relating the force acting on a single bond to macroscopic phenomena, such as acceleration of the reaction rate. In addition, FM-FDA can be combined with MD simulations, which allow the exploration of the free energy space instead of the static potential energy surface explored by COGEF- and EFEI-based simulations. The compliance-based restoring force method requires choosing an internal coordinate within the macrocycle as a constrained coordinate, which is nontrivial. In contrast, FM-FDA does not make any assumption of how the macrocycle exerts mechanical force on the reactive moiety. Additionally, although the restoring force quantifies the overall effect of external stress and is related directly to rate acceleration, FMFDA complements these insights by identifying its components on individual coordinates, including atomic interactions between both bonded and nonbonded atoms. However, FMFDA requires $M D$ simulations on long timescales (at the ns scale in this case), which limits its usage to simulations in the semiempirical or lower level for systems of tens or hundreds of atoms.

Pulling the reactive cyclobutene ring by a constant force has resulted in a symmetric distribution of internal forces, which perfectly scaled with the external force. This force distribution is fully explained by a simple model based on classical structural mechanics. It indicated that the force distribution pattern was solely determined by the mechanical geometry of the reactive moiety when an external force was applied without any connecting lever arms attached to the molecule. In contrast, FM-FDA revealed that the pulling force from photoisomerization of stiff stilbene resulted in a heterogeneous and asymmetric distribution of forces on individual bonds, which strongly depended on the properties of molecular parts other than the reactive moiety, such as the linkers and stiff stilbene. The observation of an asymmetric force distribution in asymmetric macromolecules can potentially aid the design of structurally asymmetric reactive molecule or linking polymers to selectively distribute forces onto and break bonds other than conventionally observed scissile bonds under external force. The mechanical effect of strained macrocycles on the ring opening of $t D C B$ was decomposed into three major components: the breakage of the $C B-C E$ bond and the rotation of two angles (approximated by $C A-C E$ and $C B-C F$ distances), each of which contributed mechanical work to the mechanochemical reaction to a similar extent. Despite the asymmetry of the internal force distribution, forces within critical atomic pair interactions, most importantly the scissile bond, correlated well with the effective force or acceleration of ring opening; this indicated that asymmetry played a minor role. However, interestingly, as evident from Figure 6, macrocycles with symmetric linkers, for example, $1 E, 8 E$, and $9 E$, fell in a region below the asymmetric ones for a correlation between the overall mechanical work on tDCB 
and the sum of work contributions from the three critical bonds identified. Apparently, the high stresses that propagate through primarily the short $\mathrm{X}$ linker of asymmetric macrocycles (Figure 1) primes tDCB to open more readily, or at overall smaller contributions from individual bonds. These insights could help with the design of molecular force probes for future experiments.

Thus, FM-FDA allows us to quantitatively estimate force concentration within specific bonds, and as such can help in the design of molecular force probes to funnel stresses into bonds that shall be selectively cleaved. Similarly, we expect FM-FDA to be helpful in the identification of the coordinate(s) along which mechanical force could regulate or direct the reaction to a desired product. Although the notion of molecular stress, such as ring stress in cyclic compounds ${ }^{[16,19,49-52]}$ or steric stress in inorganic complexes or proteins, ${ }^{[53-55]}$ is commonly employed to explain reactivity, FM-FDA allows, to the best of our knowledge for the first time, the quantification of such molecular stress and reveals its sources.

\section{Computational Methods}

\section{Quantum Mechanical (QM) Simulations}

As shown in Figure 1, we set up two simulation systems. System A was tDCB with its two carbon ends pulled by a constant force, thereby mimicking cyclobutene cleavage by ultrasound ${ }_{,}^{[9]}$ and system $B$ comprised of $t D C B$ inserted into macrocycles with different linkers used in molecular force probes. ${ }^{[19]}$ The structure of $t D C B$ was built by using GaussView and optimized at the B3LYP $\left(6-31 \mathrm{G}^{*}\right)$ level in Gaussian 03. ${ }^{[56]}$ The resulting structure was used as the initial structure for system A. For system B, the initial structures of macrocycles were taken from ref. [19], which were obtained by optimization at the O3LYP/6-311G $(2 d, p)$ level.

All simulations were carried out in vacuum with a modified version of the software suite GROMACS-4.0.7, ${ }^{[57]}$ which allowed us to perform QM simulations of systems A and B at the SCC-DFTB level. Stochastic dynamics (SD) simulations were performed at a temperature of $T=300 \mathrm{~K}$ and a friction coefficient of $0.5 \mathrm{ps}^{-1}$. No pressure coupling was used. The integration time step was $1 \mathrm{fs}$. For both systems A and B, after an energy minimization of 1000 steps using the steepest descent algorithm, the resulting structures were heated to $300 \mathrm{~K}$ and equilibrated for $20 \mathrm{ps}$. Then, for system $\mathrm{A}$ $2 \mathrm{~ns}$, and for system B $200 \mathrm{ps,} \mathrm{of} \mathrm{equilibrium} \mathrm{SD} \mathrm{simulations} \mathrm{were}$ performed, from which 10 structures were taken as starting structures for simulations for FM-FDA, each 2 ns for system $A$ and 1 ns for system $B$. From these production runs, starting structures were taken for conformational flooding simulations for rate estimations (details are given in Tables S1 and S3), in which molecular simulations (MD) were performed at $T=300 \mathrm{~K}$, by using a Nosé-Hoover thermostat ${ }^{[58,59]}$ with a time constant of $\tau_{\mathrm{t}}=0.5 \mathrm{ps}$.

In system $B$, only the $Z$ and $E$ isomers (-) of macrocycles $\mathbf{1}, \mathbf{4}, \mathbf{5}, \mathbf{6}$, $7, \mathbf{8}$, and $\mathbf{9}$ were simulated ${ }^{[19]}$ (Figure $1 \mathrm{~B}$ ). Due to the lack of parameters for sulfur elements in SCC-DFTB, macrocycles $\mathbf{2}$ and 3, which contained sulfur atoms, were not included in this study.

\section{Force Matching-Force Distribution Analysis (FM-FDA)}

\section{Force Distribution Analysis}

Conventional FDA was developed to analyze the change in internal forces when the system was under external perturbations, which could be, for example, an external pulling force. ${ }^{[34,60]}$ Because the force on an atom averaged to zero over time, pairwise forces between atoms were used to evaluate internal stress upon the external perturbation. The difference in the pairwise force between atoms $\mathrm{i}$ and $\mathrm{j}, \Delta F_{\mathrm{ij}}$, after and before the application of force was monitored [Eq. (3)]:

$\Delta F_{\mathrm{ij}}=F_{\mathrm{ij}}^{\text {pert }}-F_{\mathrm{ij}}^{\text {unpert }}$

in which $F_{\mathrm{ij}}^{\text {unpert }}$ is the unperturbed state prior to force application and $F_{i j}^{\text {pert }}$ is the perturbed state after the application of force; a force of one magnitude smaller than that leading to ring opening on the nanosecond timescale of our simulations. Pairwise forces were averaged over the whole simulation time for each of the unperturbed and perturbed states. Standard MD simulations were performed for this purpose, without the application of a flooding potential.

In conventional FDA, the pairwise forces can be directly extracted from the nonpolarizable force field. For QM systems, in contrast, FDA cannot be applied to extract the pairwise forces, and we instead propose an approach based on force matching. ${ }^{[37]}$

\section{Force Matching}

Force matching was proposed by Ercolessi and Adams to construct an effective force field from ab initio molecular dynamics (AIMD) simulations. ${ }^{[37]}$ The method was then largely extended and improved by Voth and co-workers ${ }^{[61-63]}$ into the multiscale coarsegraining (MS-CG) method, and they also developed a force matching scheme, MSCGFM, for MS-CG computations. ${ }^{[61,64]}$ In MS-CG, an effective force field, defined by a set of $L$ parameters $\alpha_{1}, \alpha_{2}, \ldots, \alpha_{L}$, is optimized by minimizing the differences between the forces supplied by AIMD simulations and those obtained by the effective force field. For a trajectory with $M$ configurations and $N$ atoms in each configuration, matching was achieved by minimizing the objective function given by Equation (4):

$\chi^{2}=\frac{1}{3 \mathrm{MN}} \sum_{\mathrm{i}=1}^{\mathrm{N}} \sum_{\mathrm{j}=1}^{\mathrm{M}}\left|F_{\mathrm{ij}}^{\mathrm{ref}}-F_{\mathrm{ij}}^{\mathrm{mat}}\left(\alpha_{1}, \alpha_{2} \ldots, \alpha_{\mathrm{L}}\right)\right|^{2}$

in which $F_{i j}^{\text {ref }}$ is the atomic force on atom $\mathrm{i}$ in configuration $\mathrm{j}$ obtained from AIMD simulations and serves as a reference, and $F_{\mathrm{ij}}^{\text {mat }}$ is the matched force estimated by the effective force field. Generally, MN is much larger than $L$; therefore, the number of equations is larger than the number of variables that need to be determined. Thus, Equation (4) is overdetermined and solved in the leastsquared sense.

\section{Combination of FDA and FM}

In AIMD simulations, the electronic Schrödinger equation is solved for every step of the MD simulation. Because the electrons are delocalized and correlated, it is not trivial to approximate the quantum Hamiltonian by classical pairwise interactions between atoms. Herein, we applied force matching to construct a specific PWFF from the results of quantum simulations, and thus, the resulting PWFF was an approximation of the quantum Hamiltonian. Then, 
conventional FDA was applied to analyze the trajectories from AIMD simulations with the specific PWFF. This procedure combined FDA and FM, and enabled us to perform FDA in a quantum chemical system, although in an indirect way of force matching, referred to herein as FM-FDA.

The constructed PWFF comprised of two-body (pairwise) interactions, which could be bonded and nonbonded interactions. In the bonded interactions, only 1-2, 1-3, and 1-4 two-body interactions were included, which corresponded to bond, angle, and dihedral interactions. ${ }^{[64]}$ Herein, angle and dihedral interactions were approximated by interactions between their two end atoms, that is, 1-3 and 1-4 two-body interactions, respectively. Instead of treating electrostatic interactions between charged atoms individually, the nonbonded interactions were described by a single term of interaction, which combined van der Waals and Coulombic interactions. Force matching to obtain the parameters for this PWFF was performed by using the MSCGFM software. ${ }^{[61,64]}$ The computational details of the MSCGFM are given in the Supporting Information.

\section{Conformational Flooding}

Conventional MD simulations cannot tackle rare events, such as chemical reactions or slow conformational motions of macromolecules. Conformational flooding ${ }^{[42,43]}$ has thus been developed to accelerate the simulation of such rare events by introducing a flooding potential, which is built based on the simulation of one metastable state [conformational substates (CSs)], for example, the RS of a chemical reaction. The flooding potential adopts a shape similar to that of the free energy surface of CSs and is thus able to drive the system out of the CSs and accelerate sampling without bias. The barrier of the effective flooding potential can be adjusted to modulate the extent of acceleration, which can be quantified by the increase in free energy of the $\mathrm{CS}$, which is the destabilization free energy $\Delta G^{\mathrm{fl}}$. Hence, the rate with flooding potential, $k_{\mathrm{fl}}$, is given by Equation (5):

$k_{\mathrm{fl}}=k_{0} \cdot \exp \left(\Delta G^{\mathrm{fl}} / k_{\mathrm{B}} T\right)$

in which $k_{0}$ is the rate without flooding potential, $k_{\mathrm{B}}$ is the Boltzmann constant, and $T$ is the absolute temperature. Details are given in the Supporting Information.

\section{Acknowledgements}

We thank Carsten Kutzner for technical help and Gregory A. Voth for permission to use MSCGFM. We thank the Klaus Tschira Foundation and the DAAD Sino-German Junior Research Group program for financial support. W.L. is grateful for an MPG-CAS Ph.D. scholarship.

Keywords: computational chemistry - conformation analysis cyclobutenes · force distributions · mechanochemistry

[1] M. K. Beyer, H. Clausen-Schaumann, Chem. Rev. 2005, 105, $2921-2948$

[2] M. M. Caruso, D. A. Davis, Q. Shen, S. A. Odom, N. R. Sottos, S. R. White, J. S. Moore, Chem. Rev. 2009, 109, 5755.

[3] A. L. Black, J. M. Lenhardt, S. L. Craig, J. Mater. Chem. 2011, 21, 1655.

[4] Z. Huang, R. Boulatov, Chem. Soc. Rev. 2011, 40, 2359.

[5] J. Ribas-Arino, D. Marx, Chem. Rev. 2012, 112, 5412.

[6] T. Nguyen, H. H. Kausch, Macromol. Synth. Order Adv. Prop. 1992, 100, 73.
[7] G. Cravotto, P. Cintas, Chem. Sci. 2012, 3, 295.

[8] S. S. Sheiko, F. C. Sun, A. Randall, D. Shirvanyants, M. Rubinstein, H. Lee, K. Matyjaszewski, Nature 2006, 440, 191.

[9] C. R. Hickenboth, J. S. Moore, S. R. White, N. R. Sottos, J. Baudry, S. R. Wilson, Nature 2007, 446, 423.

[10] J. N. Brantley, K. M. Wiggins, C. W. Bielawski, Science 2011, 333, 1606.

[11] J. M. Lenhardt, M. T. Ong, R. Choe, C. R. Evenhuis, T. J. Martinez, S. L. Craig, Science 2010, 329, 1057

[12] A. P. Wiita, R. Perez-Jimenez, K. A. Walther, F. Graeter, B. J. Berne, A. Holmgren, J. M. Sanchez-Ruiz, J. M. Fernandez, Nature 2007, 450, 124127.

[13] S. R. K. Ainavarapu, A. P. Wiita, L. Dougan, E. Uggerud, J. M. Fernandez, J. Am. Chem. Soc. 2008, 130, 6479-6487.

[14] R. Perez-Jimenez, J. Li, P. Kosuri, I. Sanchez-Romero, A. P. Wiita, D. Rodriguez-Larrea, A. Chueca, A. Holmgren, A. Miranda-Vizuete, K. Becker, S. H. Cho, J. Beckwith, E. Gelhaye, J. P. Jacquot, E. A. Gaucher, E. Gaucher, J. M. Sanchez-Ruiz, B. J. Berne, J. M. Fernandez, Nat. Struct. Mol. Biol. 2009, 16, 890-896.

[15] R. Perez-Jimenez, A. Inglés-Prieto, Z. M. Zhao, I. Sanchez-Romero, J. Alegre-Cebollada, P. Kosuri, S. Garcia-Manyes, T. J. Kappock, M. Tanokura, A. Holmgren, J. M. Sanchez-Ruiz, E. Gaucher, J. M. Fernandez, Nat. Struct. Mol. Biol. 2011, 18, 592.

[16] H. M. Klukovich, T. B. Kouznetsova, Z. S. Kean, J. M. Lenhardt, S. L. Craig, Nat. Chem. 2013, 5, 110.

[17] I. Park, S. S. Sheiko, A. Nese, K. Matyjaszewski, Macromolecules 2009, 42, 1805.

[18] Y. Li, A. Nese, N. V. Lebedeva, T. Davis, K. Matyjaszewski, S. S. Sheiko, J. Am. Chem. Soc. 2011, 133, 17479.

[19] Q. Z. Yang, Z. Huang, T. J. Kucharski, D. Khvostichenko, J. Chen, R. Boulatov, Nat. Nanotechnol. 2009, 4, 302.

[20] T. J. Kucharski, Z. Huang, Q. Z. Yang, Y. Tian, N. C. Rubin, C. D. Concepcion, R. Boulatov, Angew. Chem. 2009, 121, 7174; Angew. Chem. Int. Ed. 2009, 48, 7040 .

[21] T. J. Kucharski, Q. Z. Yang, Y. Tian, R. Boulatov, J. Phys. Chem. Lett. 2010 $1,2820$.

[22] T. J. Kucharski, R. Boulatov, J. Mater. Chem. 2011, 21, 8237.

[23] J. Ribas-Arino, M. Shiga, D. Marx, Angew. Chem. 2009, 121, 4254; Angew. Chem. Int. Ed. 2009, 48, 4190.

[24] J. Ribas-Arino, M. Shiga, D. Marx, J. Am. Chem. Soc. 2010, 132, 10609.

[25] W. Li, F. Gräter, J. Am. Chem. Soc. 2010, 132, 16790.

[26] M. T. Ong, J. Leiding, H. Tao, A. M. Virshup, T. J. Martínez, J. Am. Chem. Soc. 2009, 131, 6377.

[27] G. S. Kochhar, A. Bailey, N. J. Mosey, Angew. Chem. 2010, 122, 7614; Angew. Chem. Int. Ed. 2010, 49, 7452.

[28] M. J. Kryger, A. M. Munaretto, J. S. Moore, J. Am. Chem. Soc. 2011, 133, 18992.

[29] Y. Tian, R. Boulatov, ChemPhysChem 2012, 13, 2277.

[30] Y. Tian, R. Boulatov, Chem. Commun. 2013, 49, 4187-4189.

[31] H. S. Smalø, E. Uggerud, Chem. Commun. 2012, 48, 10443.

[32] S. Akbulatov, Y. Tian, R. Boulatov, J. Am. Chem. Soc. 2012, 134, 7620

[33] R. Boulatov, Nat. Chem. 2012, 5, 84.

[34] W. Stacklies, M. C. Vega, M. Wilmanns, F. Gräter, PLoS Comput. Biol. 2009, 5, e1000306.

[35] S. Xiao, W. Stacklies, M. Cetinkaya, B. Markert, F. Gräter, Biophys. J. 2009, 96, 3997.

[36] C. Baldauf, R. Schneppenheim, W. Stacklies, T. Obser, A. Pieconka, S. Schneppenheim, U. Budde, J. Zhou, F. Gräter, J. Thromb. Haemostasis 2009, 7, 2096.

[37] F. Ercolessi, J. B. Adams, Europhys. Lett. 1994, 26, 583.

[38] P. Dopieralski, J. Ribas-Arino, D. Marx, Angew. Chem. 2011, 123, 7243; Angew. Chem. Int. Ed. 2011, 50, 7105.

[39] H. Grubmüller, B. Heymann, P. Tavan, Science 1996, 271, 997.

[40] B. Heymann, H. Grubmüller, Chem. Phys. Lett. 1999, 307, 425.

[41] B. Isralewitz, M. Gao, K. Schulten, Curr. Opin. Struc. Biol. 2001, 11, 224

[42] H. Grubmüller, Phys. Rev. E 1995, 52, 2893.

[43] O. F. Lange, L. V. Schäfer, H. Grubmüller, J. Comput. Chem. 2006, 27, 1693.

[44] M. Elstner, D. Porezag, G. Jungnickel, J. Elsner, M. Haugk, T. Frauenheim, S. Suhai, G. Seifert, Phys. Rev. B 1998, 58, 7260.

[45] O. K. Dudko, G. Hummer, A. Szabo, Phys. Rev. Lett. 2006, 96, 108101.

[46] G. Hummer, A. Szabo, Biophys. J. 2003, 85, 5. 
[47] L. Deng, T. Ziegler, J. Phys. Chem. 1995, 99, 612

[48] M. K. Beyer, J. Chem. Phys. 2000, 112, 7307.

[49] S. P. Verevkin, V. N. Emel'yanenko, A. A. Pimerzin, E. E. Vishnevskaya, J. Phys. Chem. A 2011, 115, 1992.

[50] B. Tan, X. Long, J. Li, F. Nie, J. Huang, J. Mol. Model. 2012, 18, 5127 5132.

[51] F. Agapito, P. M. Nunes, B. J. C. Cabral, R. M. B. dos Santos, J. A. M. Simões, J. Org. Chem. 2008, 73, 6213.

[52] A. R. A. S. Deshmukh, B. M. Bhawal, D. Krishnaswamy, V. V. Govande, B. A. Shinkre, A. Jayanthi, Curr. Med. Chem. 2004, 11, 1889.

[53] I. Pápai, A. Hamza, P. M. Pihko, R. K. Wierenga, Chem. Eur. J. 2011, 17 2859.

[54] C. H. Suresh, A. M. Vargheese, K. P. Vijayalakshmi, N. Mohan, N. Koga, J. Comput. Chem. 2008, 29, 1840.

[55] B. H. M. Mooers, D. E. Tronrud, B.W. Matthews, Protein Sci. 2009, 18, 863

[56] Gaussian 03, Revision C.02, M. J. Frisch, G. W. Trucks, H. B. Schlegel, G. E. Scuseria, M. A. Robb, J. R. Cheeseman, J. A. Montgomery, Jr., T. Vreven, K. N. Kudin, J. C. Burant, J. M. Millam, S. S. Iyengar, J. Tomasi, V. Barone, B. Mennucci, M. Cossi, G. Scalmani, N. Rega, G. A. Petersson, H. Nakatsuji, M. Hada, M. Ehara, K. Toyota, R. Fukuda, J. Hasegawa, M. Ishida, T. Nakajima, Y. Honda, O. Kitao, H. Nakai, M. Klene, X. Li, J. E. Knox, H. P. Hratchian, J. B. Cross, C. Adamo, J. Jaramillo, R. Gomperts, R. E. Stratmann, O. Yazyev, A. J. Austin, R. Cammi, C. Pomelli, J. W. Ochterski, P. Y. Ayala, K. Morokuma, G. A. Voth, P. Salvador, J. J. Dannenberg, G. Zak- rzewski, S. Dapprich, A. D. Daniels, M. C. Strain, O. Farkas, D. K. Malick A. D. Rabuck, K. Raghavachari, J. B. Foresman, J. V. Ortiz, Q. Cui, A. G. Baboul, S. Clifford, J. Cioslowski, B. B. Stefanov, G. Liu, A. Liashenko, P. Piskorz, I. Komaromi, R. L. Martin, D. J. Fox, T. Keith, M. A. Al-Laham, C. Y. Peng, A. Nanayakkara, M. Challacombe, P. M. W. Gill, B. Johnson, W. Chen, M. W. Wong, C. Gonzalez, J. A. Pople, Gaussian, Inc., Pittsburgh, PA, 2004.

[57] B. Hess, C. Kutzner, D. van der Spoel, E. Lindahl, J. Chem. Theory Comput 2008, 4, 435.

[58] S. Nosé, Mol. Phys. 1984, 52, 255.

[59] W. G. Hoover, Phys. Rev. A 1985, 31, 1695.

[60] W. Stacklies, C. Seifert, F. Graeter, BMC Bioinf. 2011, 12, 101.

[61] S. Izvekov, M. Parrinello, C. J. Burnham, G. A. Voth, J. Chem. Phys. 2004, 120,10896

[62] S. Izvekov, G. A. Voth, J. Phys. Chem. B 2005, 109, 2469

[63] W. G. Noid, J. W. Chu, G. S. Ayton, V. Krishna, S. Izvekov, G. A. Voth, A. Das, H. C. Andersen, J. Chem. Phys. 2008, 128, 244114.

[64] L. Lu, S. Izvekov, A. Das, H. C. Andersen, G. A. Voth, J. Chem. Theory Comput. 2010, 6, 954

Received: March 13, 2013

Revised: June 6, 2013

Published online on July 10, 2013 